\title{
Alpha-mannosidosis: correlation between phenotype, genotype and mutant MAN2B1 subcellular localisation
}

Line Borgwardt ${ }^{1 *}$, Hilde Monica Frostad Riise Stensland ${ }^{2}$, Klaus Juul Olsen ${ }^{3}$, Flemming Wibrand ${ }^{1}$, Helle Bagterp Klenow ${ }^{2}$, Michael Beck ${ }^{4}$, Yasmina Amraoui ${ }^{4}$, Laila Arash ${ }^{4}$, Jens Fogh ${ }^{5}$, Øivind Nilssen ${ }^{2,6}$, Christine I Dali ${ }^{1}$ and Allan Meldgaard Lund ${ }^{1}$

\begin{abstract}
Background: Alpha-mannosidosis is caused by mutations in MAN2B1, leading to loss of lysosomal alpha-mannosidase activity. Symptoms include intellectual disabilities, hearing impairment, motor function disturbances, facial coarsening and musculoskeletal abnormalities.

Methods: To study the genotype-phenotype relationship for alpha-mannosidosis 66 patients were included. Based on the predicted effect of the mutations and the subcellular localisation of mutant MAN2B1 in cultured cells, the patients were divided into three subgroups.

Clinical and biochemical data were collected. Correlation analyses between each of the three subgroups of genotype/ subcellular localisation and the clinical and biochemical data were done to investigate the potential relationship between genotype and phenotype in alpha-mannosidosis.

Statistical analyses were performed using the SPSS software. Analyses of covariance were performed to describe the genotype-phenotype correlations. The phenotype parameters were modelled by the mutation group and age as a covariate. P values of $<0.05$ were considered as statistically significant.

Results: Complete MAN2B1 genotypes were established for all patients. We found significantly higher scores in the Leiter-R test, lower concentrations of CSF-oligosaccharides, higher point scores in the Bruininks-Oseretsky Test of Motor Proficiency subtests (BOT-2); Upper limb coordination and Balance, and a higher FVC\% in patients in subgroup 3, harbouring at least one variant that allows localisation of the mutant MAN2B1 protein to the lysosomes compared to subgrou 2 and/or subgroup 1 with no lysosomal localization of the mutant MAN2B1 protein.

Conclusion: Our results indicate a correlation between the MAN2B1 genotypes and the cognitive function, upper limb coordination, balance, FVC\% and the storage of oligosaccharides in CSF. This correlation depends on the subcellular localisation of the mutant MAN2B1 protein.
\end{abstract}

Keywords: Alpha-mannosidosis, MAN2B1, Genotype-phenotype correlation, CNS involvement

\section{Background}

Alpha-mannosidosis is caused by pathogenic sequence variants in MAN2B1, leading to loss of lysosomal alphamannosidase activity. There is a broad phenotypic variation of manifestations, including intellectual disabilities, hearing impairment, motor function disturbances, facial

\footnotetext{
* Correspondence: line.gutte.borgwardt@regionh.dk

'Department of Clinical Genetics, Centre for Inherited Metabolic Diseases, Copenhagen University Hospital Rigshospitalet, 9 Blegdamsvej, 2100 Copenhagen, Denmark

Full list of author information is available at the end of the article
}

coarsening, musculoskeletal abnormalities, and immune deficiency $[1,2]$.

Together with other lysosomal exoglycosidases, alphamannosidase cleaves sugar chains in a specific sequence as a part of the glycoprotein degradation. After degradation, the components of the sugar chains are exported from the lysosomes into the cytosol and reutilized [3]. Alpha-mannosidase deficiency causes a block in the degradation of glycoproteins and thereby a progressive lysosomal accumulation of soluble mannose-rich 
oligosaccharides in all tissues, resulting in impaired cellular function and apoptosis [4].

MAN2B1 comprises 24 exons and encodes a 1011 amino acid polypeptide [5]. The MAN2B1 polypeptide is post-translationally modified in the endoplasmic reticulum (ER), and during maturation and endosomal transport of MAN2B1 to the lysosomes it is proteolytically cleaved into three major polypeptides named "abc", "d" and "e" of 70, 42 and $15 \mathrm{kDa}$, respectively [6]. Further specific, processing of the $70 \mathrm{kDa}$ subunit results in a total of five different polypeptides [7].

Depending on the causative MAN2B1 mutation, mutant MAN2B1 proteins have been detected in subcellular compartments such as ER and lysosomes. For instance, the protein can be folded incorrectly and arrested in the ER, or it can be folded correctly and transported to the lysosomes in an inactive form $[8,9]$.

A total of 127 MAN2B1 disease-associated mutations have been reported (HGMD ${ }^{\circ}$ Professional 2015.1 [10]. The mutations are scattered throughout the coding region and include missense mutations, nonsense mutations, frameshifting small insertions/duplications/deletions, in-frame duplications, intronic splice site mutations and large deletions. In a recent study, 96 alpha-mannosidosis-associated mutations were reported in 130 unrelated patients from 30 countries [11]. Most of these mutations were private, but three mutations, c.2248C $>\mathrm{T}$ (p.Arg750Trp), c.1830+1G $>\mathrm{C}$ and c.2426 $\mathrm{T}>\mathrm{C}$ (p.Leu809Pro), were relatively frequent, and accounted for approximately $27 \%, 5 \%$ and $3 \%$, respectively, of the disease alleles [11].

At present, there is no clear relationship between genotype and severity of the disease. The phenotypic variability is high, even between siblings with identical genotypes [ 1 , 12-15]. The molecular basis of alpha-mannosidase deficiency and the phenotype has not previously been studied systematically. However, results from two studies $[11,16]$ indicated that there was no apparent correlation between mutations and clinical phenotypes.

To study the genotype-phenotype correlation for alpha-mannosidosis further, we performed mutation analysis and investigated the potential relationship between the consequences of MAN2B1 mutations and the results of motor function tests, cognitive test, and biochemical tests, including alpha-mannosidase activity for each of the 66 patients included in the study.

\section{Materials and methods}

Data presented in this paper are based on baseline data from rhLAMAN-01; a natural history study of alphamannosidosis and on baseline data from two randomised clinical trials studying the efficacy and safety of enzyme replacement therapy (ERT) with a recombinant human alpha-mannosidase for patients with alpha-mannosidosis (rhLAMAN-02 (EudraCT number: 2010-022084-36) [17,
18]; and rhLAMAN-05 (EudraCT number: 2012000979-17) (unpublished data).

\section{Patients}

66 patients (57 unrelated) with clinically and enzymatically confirmed alpha-mannosidosis, age 5-42, were included. The majority of the patients were from Europe (60), four originated from North Africa and two from Pakistan. All patients included had the attenuated form of alpha-mannosidosis (type II). 45 patients had previously been included in rhLAMAN-01 [18], and 35 patients in the rhLAMAN-02 or rhLAMAN-05 studies.

The clinical trials were performed in compliance with the principles of the Declaration of Helsinki, ICH GCP guidelines.

\section{Mutation analysis of the MAN2B1 gene}

Mutation analysis of the MAN2B1 gene and determination of the subcellular localisation of mutant MAN2B1 protein were performed as described in Riise Stensland et al. [11]. Briefly, the 24 MAN2B1 exons, corresponding exon-intron borders and parts of the 5'- and 3'- untranslated regions were sequenced using the Sanger method. When possible, parents were analysed for the mutations found in their children in order to confirm their carrier status and the allelic phase of the mutations. The software Alamut Visual version 2.5-1 (Interactive Biosoftware) was used to aid in the interpretiation of novel variants. The effect of novel (potential) splice-site mutations was studied on cDNA synthesized from RNA isolated from peripheral blood cells. The effect of novel missense mutations was studied by expression in cultured cells as described in Riise Stensland et al. [11] and slightly modified from Kuokkanen et al. [6]. Briefly, the mutant $M A N 2 B 1$ mutations were constructed by sitedirected mutagenesis of the WT MAN2B1 cDNA inserted into the pcDNA3.1- vector and expressed in COS-7 cells and/or HeLa cells. The mutant MAN2B1 protein variants were assayed for MAN2B1 activity and the subcellular processing was determined by western blot using rabbit anti-recombinant human MAN2B1 antibodies. For subcellular localisation, HeLa-cells were grown in 8-well micro-slide chambers (Ibidi, Germany), fixated in ice-cold methanol, blocked in PBS with $0.5 \%$ BSA and stained using rabbit anti-denatured bovine MAN2B1, mouse antiLAMP1 and mouse anti-PDI (primary antibodies) and Alexa 488 goat anti-rabbit and Alexa 568 goat anti-mouse as secondary antibodies. A confocal microscope (Zeiss LSM780, Carl Zeiss Microscopy GmbH, Germany) was used to capture Z-stack images of the transfected cells.

Based on the results from the mutation analysis and predicted (null mutations) or determination of subcellular MAN2B1 protein localisation, patients were divided into three subgroups: 
I. Two null-mutations (nonsense, frameshift, large truncations). Subcellular localisation of the mutant MAN2B1 proteins was not studied.

II. At least one missense mutation (or in-frame deletion/ duplication of 1-5 amino acids) with the MAN2B1 protein localised to the endoplasmic reticulum (ER) (ie: ER/ER, ER/null).

III. At least one missense mutation (or in-frame deletion/duplication of 1-5 amino acids) with the MAN2B1 protein localised to the lysosomes (lyso) (ie: lyso/lyso, lyso/ER, lyso/null).

\section{Collection of clinical and biochemical data}

RhLAMAN-01 data were collected at four European centres. RhLAMAN-02 and rhLAMAN-05 data were collected at one center. Clinical and biochemical data were collected prospectively. Assessments, including sixminutes-walk test (6-MWT), pulmonary function test, alpha-mannosidase enzyme activity and audiometry were made in all patients included in rhLAMAN-01, rhLAMAN-02 and rhLAMAN-05 $(\mathrm{n}=66)$. Assessments, including Three-minutes-stair-climb-test (3MSCT), The Bruininks-Oseretsky Test of Motor Proficiency (BOT-2), cognitive function test, Cerebral Spinal Fluid (CSF)-oligosaccharides, S-oligosaccharides and specific CSF-biomarkers (CSF-tau-protein (CSF-T-tau), CSF-Glial Fibrillary Acidic protein (CSF-GFAp), CSFNeuroFilament Light protein (CSF-NFLp)) were made only for patients included in rhLAMAN-02 and rhLAMAN-05 $(\mathrm{n}=35)$.

\section{Biochemical assessments \\ Oligosaccharides}

Lumbar puncture and blood sampling were performed for the 35 patients participating in rhLAMAN-02 and rhLAMAN-05 for measurement of CSF-oligosaccharides and serum-oligosaccharides, respectively. Oligosaccharides in CSF and serum were determined quantitatively by electrospray tandem mass spectrometry. Assays were performed at the Danish Technology Institute, Kolding, Denmark.

\section{CSF-biomarkers of neurodegeneration}

Lumbar puncture was performed for the 35 patients participating in rhLAMAN-02 and rhLAMAN-05 and measurement of CSF biomarkers, T-tau, NFLp and GFAp were done. Thirty four of 35 CSF-samples were analysed. The total volume of CSF was seven $\mathrm{ml}$. For CSF biomarkers the CSF was frozen at $-20{ }^{\circ} \mathrm{C}$, for CSF oligosaccharides at $-80^{\circ} \mathrm{C}$.

CSF T-tau was measured using an ELISA assay (INNOTEST hTau Ag, Innogenetics, Ghent, Belgium) [19]. CSF GFAP and CSF NFLp were measured using an inhouse developed sandwich ELISA [20, 21]. Determination of levels of CSF biomarkers was performed at Sahlgrenska Hospital, Göteborg, Sweden.

\section{Alpha-mannosidase activity}

Determination of alpha-mannosidase activity was done in serum in rhLAMAN-01, and in plasma in rhLAMAN-02 and rhLAMAN-05. Both determinations were performed as described by Masson and Lundblad [22].

To make the two methods comparable, enzyme activities were reported as the percentages of the mean of the reference values.

\section{Clinical assessments \\ Cognitive function test}

The cognitive function has been investigated in another study of ours [23]. Briefly, the Leiter International Performance Scale-Revised (Leiter R) was used [24-26]. The test consists of two test batteries; Visual Function and Reasoning battery and Memory and Attention battery. For each of the test batteries a measure of the overall functioning level, a total equivalent age, is obtained. All 35 patients performed the Visual Function and Reasoning battery, only 26, enrolled or screened for rhLAMAN-05, performed the Memory and Attention battery.

\section{Pulmonary function test}

Pulmonary function test measuring the forced vital capacity (FVC) (and percentage of predicted value, depending on age, size and sex (FVC\%), was performed in accordance with American Thoracic Society (ATS)-standards and The European Respiratory Society Statements (ERS) [27].

\section{Motor function assessments}

6MWT was performed in accordance with ATS-standards [28]. 3MSCT is not a standardised test, but was performed according to published guidelines [29-31]. BOT-2 is a standardised and validated test measuring fine and gross motor skills of children and adolescents [32].

\section{Audiometry}

Unaided pure-tone audiometry at frequencies $0.25,0.5$, 1,2 and $4 \mathrm{kHz}$ was carried out in accordance with ISO 8253-1 [33].

\section{Statistical analysis}

Statistical analyses were performed using the SPSS software. Analyses of covariance were performed to describe the genotype-phenotype correlations. The phenotype parameters were modelled by the mutation group and age as a covariate. $P$ values of $<0.05$ were considered as statistically significant. 


\section{Sample size calculations}

No formal sample size calculation was performed for this study. The sample size is from a statistical point of view a small number of persons, but it represents a compromise between the limited number of persons fulfilling the inclusion/exclusion criteria and the minimal amount of data which can support a possible genotype-phenotype correlation.

\section{Results}

The diagnosis of alpha-mannosidosis was established in all 66 patients (24 females and 42 males), age 5 to 42 years (mean 19.4), from 57 families based on deficiency of acid alpha-mannosidase activity in leukocytes or serum.

\section{Mutation spectrum and functional analyses of novel missense variants}

Disease-associated MAN2B1 mutations were identified in all patients. About half of the unrelated patients were homozygous (28 of 57 ). In total we detected 56 diseaseassociated variants, of which 11 were novel (see Tables 1 and 2 and Fig. 1). None of the novel mutations were present in dbSNP (http://www.ncbi.nlm.nih.gov/projects/SNP/) but c.2885G $>$ A p.(Arg962His) was present in the large database of exome sequences ExAc (Exome Aggregation Consortium (www. http://exac.broadinstitute.org/)) with a frequency of 2,47e-05 (3 alleles out of
121 374). For 43 patients parental sequencing data were collected, for 7 patients only sequencing data from the mother or the father were collected and for 16 patients the parental samples were not available.

Mutations were scattered all over the gene and included missense mutations (23, 3 novel), small duplications $(9,4$ novel), nonsense mutations $(8,1$ novel), splice site mutations $(8,1$ novel), small deletions $(6,1$ novel), one large deletion (intron 6-13) and one novel intronic variant (c.1230+5G>A).

Based on cDNA analyses in blood, the variant c.2436 $+5 \mathrm{G}>\mathrm{A}$ in intron 20 caused skipping of exon 20 and deleted $81 \mathrm{nt}$ from the transcript (r.2356_2436del81, p.Glu786_Met812del27). The effect of c.1230+5G>A in intron 9 was not clear from the cDNA analysis and requires further analyses. It was thus classified as a variant of unknown clinical significance (VUS). However, the variant was detected in two Spanish sibs, and in this family it was on the same allele (in cis) as the known disease-causing variant c.2248C $>\mathrm{T}$ (p.Arg750Trp). All nonsense mutations, frame-shifting small deletions/duplications, large truncations caused by splice site mutations and the large deletion were considered null-alleles.

In total, 23 missense mutations were detected in our patient cohort. Of these, 3 were novel; c.304G $>\mathrm{A}$ (p.Asp102Asn), c.458G>T (p.Gly153Val) and c.2885G >A (p.Arg962His). Based on transfection experiments in cell culture, MAN2B1 p.Asp102Asn and p.Gly153Val were

Table 1 Novel MAN2B1 variants detected in this study

\begin{tabular}{|c|c|c|c|c|c|}
\hline CDNA label & Location & Predicted effect on protein & Activity $^{2}$ & Processing $^{3}$ & Location $^{4}$ \\
\hline \multicolumn{6}{|c|}{ Nonsense mutations/duplications/deletions } \\
\hline c.383G $>A$ & Exon 3 & p.(Trp128Ter) & & & \\
\hline c.809dupA & Exon 6 & p.(Asp270GlufsTer54) & & & \\
\hline c.812_813dupTG & Exon 6 & p.(Leu272CysfsTer27) & & & \\
\hline c.1047_1048dupCC & Exon 8 & p.(His350ProfsTer15) & & & \\
\hline c.2675dupT & Exon 22 & p.(Arg893AlafsTer38) & & & \\
\hline c.2944_2947delCCGT & Exon 24 & p.(Pro982ThrfsTer50) & & & \\
\hline \multicolumn{6}{|l|}{ Splice site mutations } \\
\hline c. $1230+5 G>A^{1}$ & Intron 9 & p.? & & & \\
\hline$c .2436+5 G>A$ & Intron 20 & p.Glu786_Met812del & & & \\
\hline \multicolumn{6}{|l|}{ Missense mutations } \\
\hline c.304G $>A$ & Exon 3 & p.(Asp102Asn) & No & Yes & Lysosomal \\
\hline$c .458 \mathrm{G}>\mathrm{T}$ & Exon 4 & p.(Gly153Val) & No & Yes & Lysosomal \\
\hline c. $2885 G>A^{5}$ & Exon 22 & p.(Arg962His) & Yes & Yes & Lysosomal \\
\hline
\end{tabular}

Mutations were labelled following the most recent Human Genome Variation Society (HGVS) guidelines (version 2.121101, www.hgvs.org/mutnomen). Mutant residues were numbered using the MAN2B1 reference sequence NM 000528.3. The systematic names are preceded by a "c." following the HGVS recommendations for cDNA reference numbering, with +1 as A of the initiation codon ATG. At the protein level, names are preceded by "p.". Amino acids are listed according to the three-letter code. Protein labels are in parentheses if the variant has not been studied on RNA or protein level

${ }^{1}$ Located on the same allele (in cis) as the known pathogenic missense variant c.2248C $>$ T p.Arg750Trp

${ }^{2}$ In lysates of transiently transfected COS-7 cells: No $\leq 20 \%$ of WT; Some $=20-30 \%$ of WT; Yes $\geq 30 \%$ of WT

${ }^{3}$ In lysates of transiently transfected COS-7 cells and HeLa-cells

${ }^{4}$ In vivo localisation in transiently transfected HeLa-cells

${ }^{5}$ Variant of uncertain clinical significance 
Table 2 MAN2B1 mutations and genotype/subcellular localisation subgroups for all patients

\begin{tabular}{|c|c|c|c|c|c|c|}
\hline \multirow[b]{2}{*}{ Family number } & \multicolumn{2}{|l|}{ Allele 1} & \multicolumn{2}{|l|}{ Allele 2} & \multirow[t]{2}{*}{ Localisation } & \multirow{2}{*}{$\begin{array}{l}\text { Genotype group }{ }^{10} / \\
\text { subcellular localisation } \\
\text { subgroups }\end{array}$} \\
\hline & Label cDNA & Label Protein & Label cDNA & Label Protein & & \\
\hline$\overline{1 A^{1}}$ & c.164G>T & p.(Cys55Phe) & c.599A>T & p.(His200Leu) & Exon 2 / Exon 4 & 3 \\
\hline $1 B^{1}$ & c.164G>T & p.(Cys55Phe) & C.599A $>T$ & p.(His200Leu) & Exon 2 / Exon 4 & 3 \\
\hline 2 & c. $231 \mathrm{G}>\mathrm{A}$ & p.(Trp77Ter) & c. $2398 \mathrm{G}>\mathrm{C}$ & p.(Gly800Arg) & Exon 2 / Exon 20 & $2^{*}$ \\
\hline 3 & c. $283 \mathrm{G}>\mathrm{C}$ & p.(Ala95Pro) & c.283G $>C$ & p(.Ala95Pro) & Exon 3 / Exon 3 & 2 \\
\hline 4 & c. $283 G>C$ & p.(Ala95Pro) & c. $283 G>C$ & p.(Ala95Pro) & Exon 3 / Exon 3 & 2 \\
\hline 5 & c. $304 \mathrm{G}>\mathrm{A}$ & p.(Asp102Asn) & c. $2885 \mathrm{G}>\mathrm{A}$ & p.(Arg962His) & Exon 3 / Exon 23 & 3 \\
\hline 6 & c. 338_348dup11 & p.(lle117ProfsTer44) & c. $338 \_348 d u p 11$ & p.(lle117ProfsTer44) & Exon 3 / Exon 3 & 1 \\
\hline 7 & c.383G $>A$ & p.(Trp128Ter) & c.383G $>A$ & p.(Trp128Ter) & Exon 3 / Exon 3 & $1^{*}$ \\
\hline 8 & c. $418 C>T$ & p.(Arg140Ter) & c. $418 C>T$ & p.(Arg140Ter) & Exon 3 / Exon 3 & 1 \\
\hline $9 A^{2}$ & $c .458 \mathrm{G}>\mathrm{T}$ & p.(Gly153Val) & c. $[1230+5 G>A ; c .2248 C>T]$ & p.[?; Arg750Trp] & Exon 4 / Intron 9 & 3 \\
\hline $9 B^{2}$ & $c .458 \mathrm{G}>\mathrm{T}$ & p.(Gly153Va) I & c. $[1230+5 G>A ; c .2248 C>T]^{11}$ & p.[?; Arg750Trp] & Exon 4 / Intron 9 & 3 \\
\hline 10 & C. $.590 C>G$ & p.(Pro197Arg) & c. $2724 \mathrm{G}>\mathrm{A}$ & p.(Trp908Ter) & Exon 4 / Exon 22 & $2^{*}$ \\
\hline 11 & C. $.598 \mathrm{C}>\mathrm{A}$ & p.(His200Asn) & c.1548delT & p.(Leu518TrpfsTer5) & Exon 4 / Exon 13 & $3^{*}$ \\
\hline 12 & c. $685 C>T$ & p.(Arg229Trp) & c.2439_2444dup6 & p.(His814_Arg815dup) & Exon 5 / Exon 21 & $3^{*}$ \\
\hline 13 & c. $783 C>A$ & p.(Tyr261Ter) & c. $783 C>A$ & p.(Tyr261Ter) & Exon 6 / Exon 6 & 1 \\
\hline 14 & c. $788 \mathrm{C}>\mathrm{T}$ & p.Pro263Leu & c. $2355 \mathrm{G}>\mathrm{A}$ & p.(Arg757MetfsTer6) & Exon 6 / Exon 19 & 3 \\
\hline 15 & c.809dupA & p.(Asp270GlufsTer54) & c.2675dupT & p.(Arg893AlafsTer38) & Exon 6 / Exon 22 & 1 \\
\hline 16 & c.812_813dupTG & p.(Leu272CysfsTer27) & c.812_813dupTG & p.(Leu272CysfsTer27) & Exon 6 / Exon 6 & 1 \\
\hline 17 & c.909+731 del6272 & p.Gly304del245 & $c .953 C>T$ & p.Ser318Leu & Intron 6-13 / Exon 7 & 3 \\
\hline 18 & c. $1026+2 \mathrm{~T}>\mathrm{G}$ & $\begin{array}{l}\text { p.[Val339_Ala341del; } \\
\text { p.Val339_Gln342del] }\end{array}$ & C. $1830+1 G>C$ & p.Val549_Glu610del & Intron 7 / Intron 14 & 2 \\
\hline 19 & c.1047_1048dupCC & p.(His350ProfsTer15) & c. $2248 \mathrm{C}>\mathrm{T}$ & p.Arg750Trp & Exon 8 / Exon 18 & 2 \\
\hline $20 A^{3}$ & C.1055 T>C & p.(Leu352Pro) & C.1055 T>C & p.(Leu352Pro) & Exon 8 / Exon 8 & 2 \\
\hline $20 B^{3}$ & C.1055 T>C & p.(Leu352Pro) & C.1055 T>C & p.(Leu352Pro) & Exon 8 / Exon 8 & 2 \\
\hline 21 & c.1152_1153dupCC & p.(His385ProfsTer93) & c. $1831-2 A>G$ & p.His611GlyfsTer3 & Exon 9 / Intron 14 & 1 \\
\hline 22 & c.1152_1153dupCC & p.(His385ProfsTer93) & c.1152_1153dupCC & p.His385ProfsTer93 & Exon 9 / Exon 9 & 1 \\
\hline 23 & c. $1310-2 A>G$ & p.(?) & $c .2248 C>T$ & p.Arg750Trp & Intron 10 / Exon 18 & 2 \\
\hline 24 & C. $1333 C>T$ & p.(His445Tyr) & C. $1333 C>T$ & p.(His445Tyr) & Exon 11 / Exon 11 & 2 \\
\hline 25 & c.1351G>T & p.(Gly451Cys) & c.[1501 T>A; $2849 \mathrm{G}>C]^{11}$ & p.([Cys501Ser; Arg950Pro]) & Exon 11 / Exon 12 & 3 \\
\hline $26 A^{4}$ & C. $1358 \mathrm{C}>\mathrm{T}$ & p.(Ser453Phe) & c. $1358 \mathrm{C}>\mathrm{T}$ & p.(Ser453Phe) & Exon 11 / Exon 11 & 2 \\
\hline $26 B^{4}$ & C.1358C $>T$ & p.(Ser453Phe) & C.1358C >T & p.(Ser453Phe) & Exon 11 / Exon 11 & 2 \\
\hline $27 A^{5}$ & C.1370 T>A & p.(Val457Glu) & $c .2248 C>T$ & p.Arg750Trp & Exon 11 / Exon 18 & $3^{*}$ \\
\hline
\end{tabular}


Table 2 MAN2B1 mutations and genotype/subcellular localisation subgroups for all patients (Continued)

\begin{tabular}{|c|c|c|c|c|c|c|}
\hline $27 \mathrm{~B}^{5}$ & C. $1370 \mathrm{~T}>\mathrm{A}$ & p.(Val457Glu) & c. $2248 C>T$ & p.Arg750Trp & Exon 11 / Exon 18 & $3^{*}$ \\
\hline $28 A^{6}$ & C. $1383 C>A$ & p.(Tyr461Ter) & c.2402dupG & p.(Ser802GInfsTer129) & Exon 11 / Exon 20 & $1^{*}$ \\
\hline $28 B^{6}$ & C. $1383 C>A$ & p.(Tyr461Ter) & c.2402dupG & p.(Ser802GInfsTer129) & Exon 11 / Exon 20 & $1^{*}$ \\
\hline 29 & c.1388_1389delGC & p.(Arg463ProfsTer53) & c.2426 T>C & p.Leu809Pro & Exon 11 / Exon 20 & $2^{*}$ \\
\hline 30 & c. $1527+1 G>C$ & p.(?) & c. $1527+1 \mathrm{G}>\mathrm{C}$ & p.(?) & Intron 12 / Intron 12 & $1^{*}$ \\
\hline $31 A^{7}$ & c.1816delA & p.Thr606ProfsTer 18 & c. $1830+1 \mathrm{G}>\mathrm{C}$ & p.Val549_Glu610del & Exon 14 / Intron 14 & 1 \\
\hline $31 B^{7}$ & c.1816delA & p.Thr606ProfsTer18 & c. $1830+1 G>C$ & p.Val549_Glu610del & Exon 14 / Intron 14 & 1 \\
\hline 32 & c. $1830+1 G>A$ & p.(?) & c. $2248 \mathrm{C}>\mathrm{T}$ & p.Arg750Trp & Intron 14 / Exon 18 & 2 \\
\hline 33 & C. $1830+1 G>C$ & p.Val549_Glu610del & c. $1830+1 \mathrm{G}>\mathrm{C}$ & p.Val549_Glu610del & Intron 14 / Intron 14 & 1 \\
\hline 34 & C. $1830+1 G>C$ & p.Val549_Glu610del & c. $2248 C>T$ & p.Arg750Trp & Intron 14 / Exon 18 & 2 \\
\hline 35 & C. $1830+1 G>C$ & p.Val549_Glu610del & C.2426 T>C & p.Leu809Pro & Intron 14 / Exon 20 & 2 \\
\hline 36 & C. $1830+1 G>C$ & p.Val549_Glu610del & c. $2248 C>T$ & p.Arg750Trp & Intron 14 / Exon 18 & 2 \\
\hline $37 A^{8}$ & c. $1831-2 A>G$ & p.His611GlyfsTer3 & c. $1831-2 A>G$ & p.His611GlyfsTer3 & Intron 14 / Intron 14 & 1 \\
\hline $37 B^{8}$ & c. $1831-2 A>G$ & p.His611GlyfsTer3 & c. $1831-2 A>G$ & p.His611GlyfsTer3 & Intron 14 / Intron 14 & 1 \\
\hline 38 & c. $2234 C>G$ & p.(Thr745Arg) & c. $2234 C>G$ & p.Thr745Arg & Exon 18 / Exon 18 & 3 \\
\hline $39 A^{9}$ & $c .2248 C>T$ & p.Arg750Trp & c. $2248 \mathrm{C}>\mathrm{T}$ & p.Arg750Trp & Exon 18 / Exon 18 & $2^{*}$ \\
\hline $39 B^{9}$ & $c .2248 C>T$ & p.Arg750Trp & c. $2248 \mathrm{C}>\mathrm{T}$ & p.Arg750Trp & Exon 18 / Exon 18 & $2^{*}$ \\
\hline 40 & $c .2248 C>T$ & p.Arg750Trp & c. $2248 C>T$ & p.Arg750Trp & Exon 18 / Exon 18 & $2^{*}$ \\
\hline 41 & c. $2248 \mathrm{C}>\mathrm{T}$ & p.Arg750Trp & $c .2248 C>T$ & p.Arg750Trp & Exon 18 / Exon 18 & $2^{*}$ \\
\hline 42 & c. $2248 \mathrm{C}>\mathrm{T}$ & p.Arg750Trp & c. $2248 \mathrm{C}>\mathrm{T}$ & p.Arg750Trp & Exon 18 / Exon 18 & $2^{*}$ \\
\hline 43 & c. $2248 \mathrm{C}>\mathrm{T}$ & p.Arg750Trp & c. $2248 \mathrm{C}>\mathrm{T}$ & p.Arg750Trp & Exon 18 / Exon 18 & $2^{*}$ \\
\hline 44 & c. $2248 \mathrm{C}>\mathrm{T}$ & p.Arg750Trp & c. $2299 C>T$ & p.GIn767Ter & Exon 18 / Exon 19 & $2^{*}$ \\
\hline 45 & c. $2248 \mathrm{C}>\mathrm{T}$ & p.Arg750Trp & c. $2248 \mathrm{C}>\mathrm{T}$ & p.Arg750Trp & Exon 18 / Exon 18 & 2 \\
\hline 46 & $c .2248 C>T$ & p.Arg750Trp & $c .2248 C>T$ & p.Arg750Trp & Exon 18 / Exon 18 & $2^{*}$ \\
\hline 47 & $c .2248 C>T$ & p.Arg750Trp & c. $2426 \mathrm{~T}>\mathrm{C}$ & p.Leu809Pro & Exon 18 / Exon 20 & $2^{*}$ \\
\hline 48 & c. $2248 \mathrm{C}>\mathrm{T}$ & p.Arg750Trp & c. $2248 \mathrm{C}>\mathrm{T}$ & p.Arg750Trp & Exon 18 / Exon 18 & 2 \\
\hline 49 & c. $2248 \mathrm{C}>\mathrm{T}$ & p.Arg750Trp & c. $2426 \mathrm{~T}>\mathrm{C}$ & p.Leu809Pro & Exon 18 / Exon 20 & 2 \\
\hline 50 & c. $2248 C>T$ & p.Arg750Trp & c. $2251 \mathrm{G}>\mathrm{T}$ & p.(Glu751Ter) & Exon 18 / Exon 20 & $2^{*}$ \\
\hline 51 & c. $2248 \mathrm{C}>\mathrm{T}$ & p.Arg750Trp & $c .2248 C>T$ & p.Arg750Trp & Exon 18 / Exon 18 & 2 \\
\hline 52 & c. $2355 \mathrm{G}>\mathrm{A}$ & p.Arg757MetfsTer6 & c. $2355 \mathrm{G}>\mathrm{A}$ & p.(Arg757MetfsTer6) & Exon 19 / Exon 19 & 1 \\
\hline 53 & $c .2398 G>T$ & p.(Gly800Trp) & c.2944_2947delCCGT & p.(Pro982ThrfsTer50) & Exon 20 / Exon 24 & 2 \\
\hline 54 & c. $2436+5 G>A$ & p.Glu786_Met812del & c.2887delG & p.(Glu963ArgfsTer70) & Intron 20 / Exon 23 & 1 \\
\hline
\end{tabular}


Table 2 MAN2B1 mutations and genotype/subcellular localisation subgroups for all patients (Continued)

\begin{tabular}{|c|c|c|c|c|c|c|}
\hline 55 & c. $2724 G>A$ & p.(Trp908Ter) & c. $2724 \mathrm{G}>\mathrm{A}$ & p.(Trp908Ter) & Exon 22 / Exon 22 & 1 \\
\hline 56 & c.2920dupA & p.(Thr974AsnfsTer81) & c.2920dupA & p.(Thr974AsnfsTer81) & Exon 23 / Exon 23 & $1^{*}$ \\
\hline 57 & c.2921_2922delCA & p.(Thr974ArgfsTer80) & c.2921_2922delCA & p.(Thr974ArgfsTer80) & Exon 23 / Exon 23 & 1 \\
\hline
\end{tabular}

Mutations are labelled according to HGVS recommendations (http://www.hgvs.org/mutnomen/) using the MAN2B1 coding DNA reference sequence NM_000528.3, where position +1 corresponds to A in the first ATG translation initiation codon. Novel mutations are in bold. Protein labels are in parentheses if the variant has not been studied on RNA or protein level.

${ }_{1-9}$ Sibship 1-9, ${ }^{10}$ Subgroup 1: Two null-mutations (nonsense, frameshift, large truncations), subgroup 2: At least one missense mutation (or in-frame deletion/duplication of 1-5 amino acids) with the MAN2B1 protein localised to the endoplasmic reticulum (ER) (ie: ER/ER, ER/null), subgroup 3: At least one missense mutation (or in-frame deletion/duplication of 1-5 amino acids) with the MAN2B1 protein localised to the lysosomes

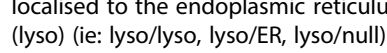

*Incomplete or no parental sequencing data available 


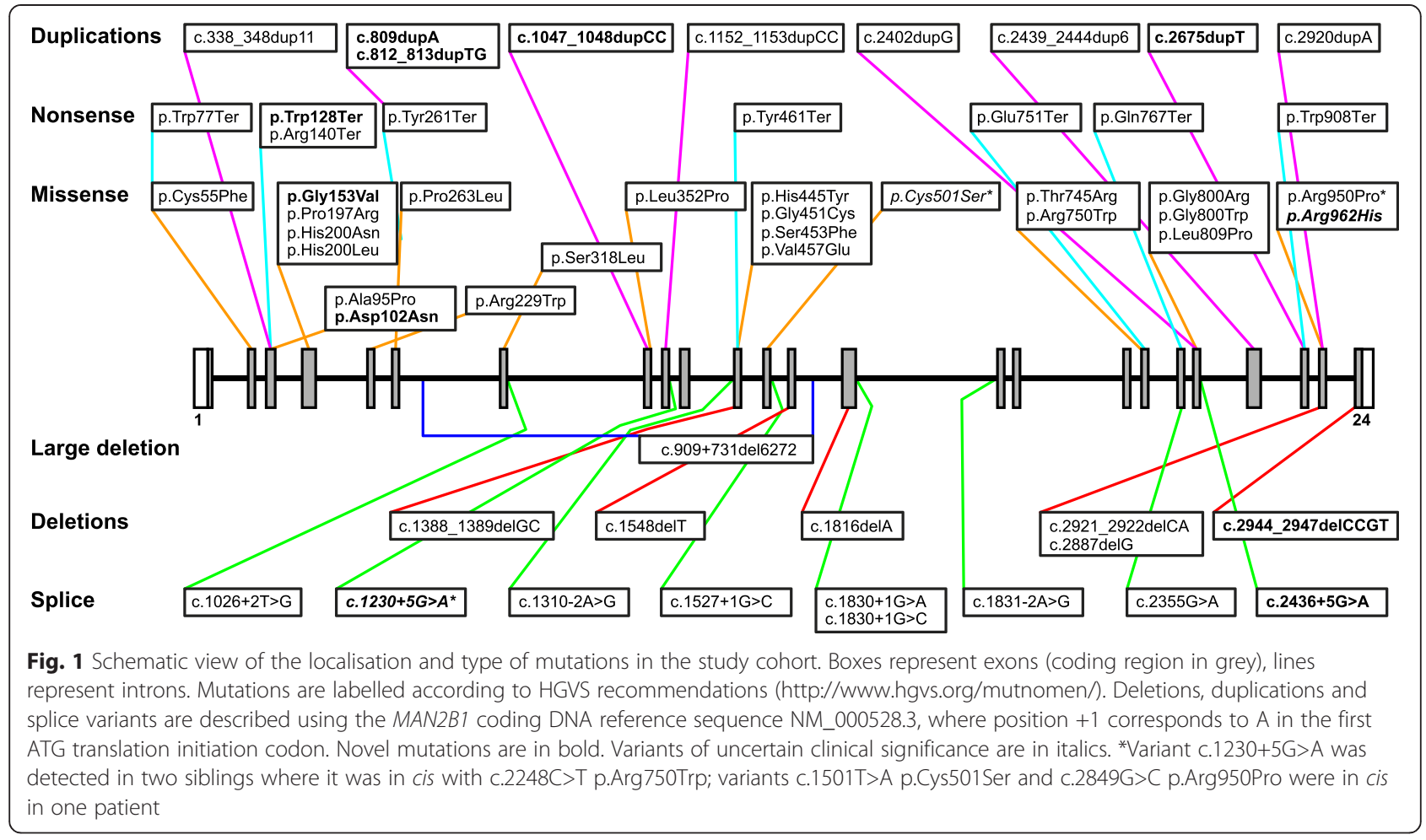

found to be inactive, processed correctly, secreted into the medium and localised to the lysosomes (Figs. 2 and 3). MAN2B1 p.Arg962His showed considerable residual activity indistinguishable from the activity observed for common, non-pathogenic variants [7] and in some experiments resembling the level of the WT, and was processed correctly and localised to the lysosomes. However, the intensity of the bands representing the

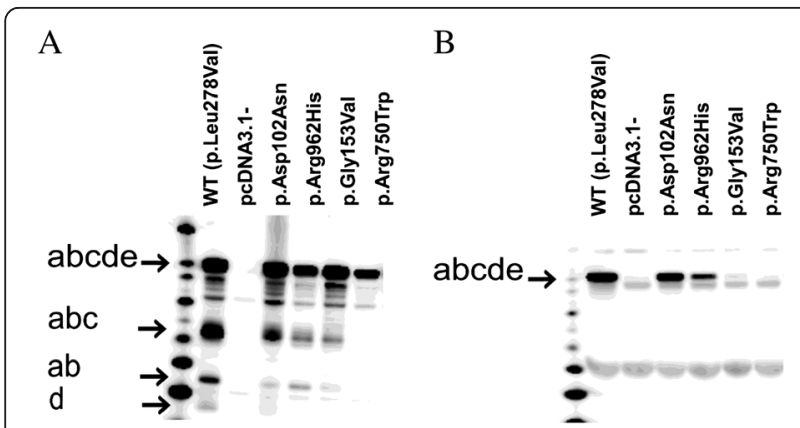

Fig. 2 Western blot showing the intracellular processing and secretion of the novel MAN2B1 missense variants in transfected COS-7 cells. The relative intensity of the different peptides is different for the wild-type enzyme and the missense variants. a. Overexpressed and transported MAN2B1 proteins are also secreted into the cell media in the full-length form. b. The WT was included as a positive control of MAN2B1 processing/secretion, pcDNA3.1 was included as a negative control of MAN2B1 expression (cells transfected with an empty vector) and MAN2B1 p.Arg750Trp was included as a negative control of MAN2B1 processing/secretion (accumulates in the ER) processed peptides (as seen on western blot) was weaker for the variant as compared to the wild-type MAN2B1 (Figs. 2 and 3), and it was consequently classified as a VUS.

\section{Genotype-phenotype correlations}

Although all the patients included in this study were classified as having the attenuated - type II alphamannosidosis, their clinical phenotypes demonstrated a large degree of variability. On the basis of the mutation analysis, and the predicted effect of the mutations, the patients were divided into the genotype/subcellular localisation subgroups. The distribution of patients and ages in the three genotype/subcellular localisation subgroups are shown in Table 3.

The correlation between the three genotype/subcellular localisation subgroups and CNS related clinical and biochemical data are shown in Fig. 4, the correlation between the three genotype/subcellular localisation subgroups and the motor function test and $\mathrm{FVC} \%$ are shown in Fig. 5.

Beside the significant correlations demonstrated in Table 4, correlations between the genotype/subcellular localisation subgroups, including pairwise comparison, and S-oligosaccharides; CSF-biomarkers; alpha-mannosidase activity (\%); 6-MWT; 3-MSCT and audiometry were performed. No significant correlations were found (S-oligosaccharides: $\mathrm{p}=0.76$ (age $\mathrm{p}=0.86$ ), CSF-GFAp: $\mathrm{p}=0.11$ (age $\mathrm{p}=0.94$ ), CSF-T-tau: $\mathrm{p}=0.12$ (age $\mathrm{p}=0.006$ ), CSF-NFLp: 

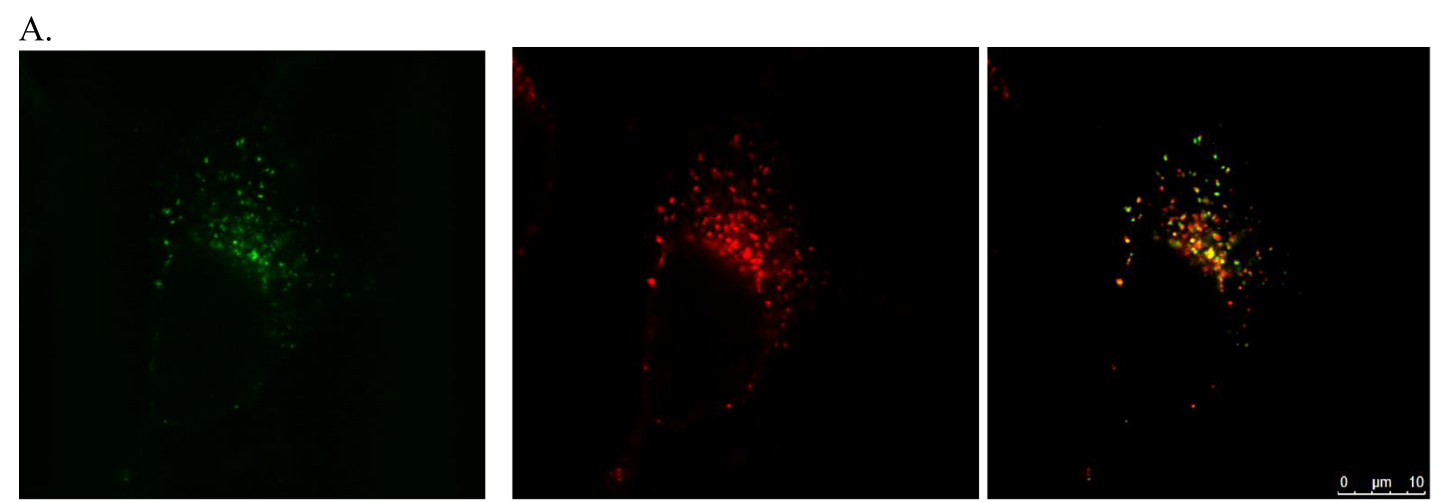

B.
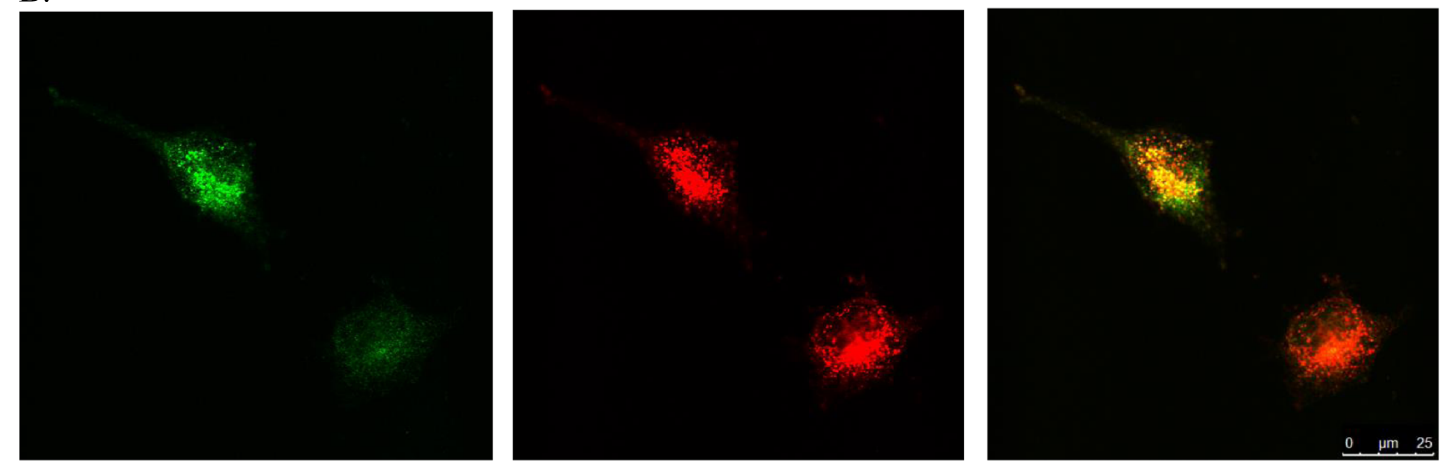

C.
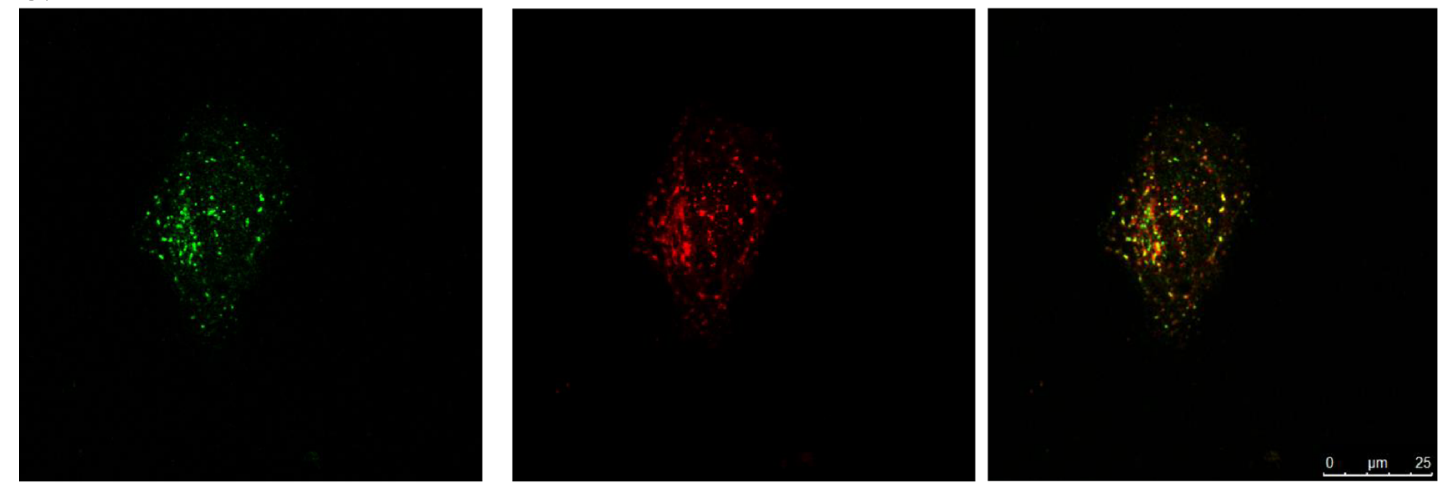

D.
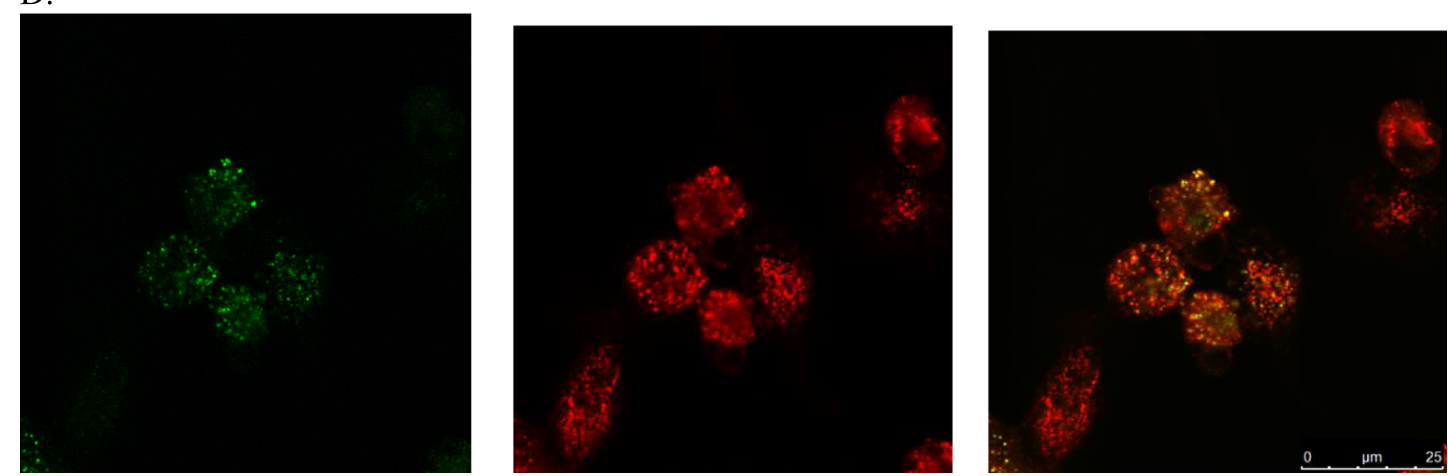

Fig. 3 (See legend on next page.) 
(See figure on previous page.)

Fig. 3 Confocal fluorescent microscopy images showing the intracellular localisation of the novel MAN2B1 missense variants in transfected HeLa-cells. The first column of images shows methanol-fixed transfected HeLa-cells stained for MAN2B1 (green), the second column shows the same cells stained for the lysosomal marker LAMP1 (red) and the third column shows merged images with colocalized MAN2B1 and LAMP1 (yellow). a: MAN2B1 p.Asp102Asn; b: MAN2B1 p.Gly153Val; c: MAN2B1 p.Arg962His; d: WT. The WT was included as a positive control of lysosomal localization

$\mathrm{p}=0.46$ (age $\mathrm{p}=0.18)$, alpha-mannosidase activity (\%): $\mathrm{p}=0.19$ (age $\mathrm{p}=0.10$ ), 6-MWT: $\mathrm{p}=0.102$ (age $\mathrm{p}=0.01$ ): 3MSCT: $\mathrm{p}=0.82$ (age $\mathrm{p}=0.60$ ), Bone conducted hearing loss: $\mathrm{p}=0.90$ (age $\mathrm{p}=0.001)$ : Air conducted hearing loss: $\mathrm{p}=0.37$ (age $\mathrm{p}=0.26$ ). Because of the non-significant results, pairwise comparisons are not reported.

\section{Discussion}

Two main findings have been obtained in this first systematic study of the potential relationship between genotype and phenotype in alpha-mannosidosis.

Firstly, by analysing 66 patients from 57 families, 56 disease-associated mutations were identified. Complete $M A N 2 B 1$ genotypes were established for all patients included.

Forty five mutations were described previously and 11 were novel. In accordance with previous findings, the mutations were scattered all over the $M A N 2 B 1$ gene and included most types of mutations. The most frequent missense mutation, c.2248C $>\mathrm{T}$ (p.Arg750Trp), was detected in 20 unrelated patients from 9 countries and accounted in this study for $25.4 \%$ of the disease alleles in unrelated patients, which is in accordance with previous reports $[11,16]$.

Secondly, we found that genotypes, allowing mutant MAN2B1 protein to enter the lysosomes, correlated positively with several clinical and biochemical parameters.

Based on the predicted severity of the genotypes and subcellular localisation of the mutant MAN2B1 protein, we classified our patients into three subgroups. We hypothesised that patients from subgroups 1 and 2, either with two null-mutations or production of incorrectly folded MAN2B1 proteins were expected to have the most severe phenotype. The patients in subgroup 3, with at least one variant that allows localisation of the mutant MAN2B1 protein to the lysosomes, were hypothesised to show the mildest clinical presentation. They were expected to produce MAN2B1 proteins which fold sufficiently to reach the lysosomes and which potentially could confer some residual MAN2B1 activity.

As hypothesised, patients from subgroup 3, harbouring at least one variant that allows localisation of the mutant MAN2B1 protein to the lysosomes, performed significantly better and had less abnormal results in some of the clinical tests and biochemical analyses, compared to patients in subgroup 2 and/or subgroup 1. Thus, in patients from subgroup 3 , we found a significantly higher total equivalent age in the two batteries employed in the cognitive test, a higher point score in two BOT-2 subtests (Upper limb coordination and Balance), a higher FVC\% and lower concentrations of CSF-oligosaccharides compared to the other groups.

Our findings indicate a correlation between genotype/ intercellular localisation and the impact on CNS, pulmonary function and motor function. The genotypephenotype correlation concerning the CNS function, are supported by a significant negative correlation between level of CSF-oligosaccharides and level of cognitive function in the same cohort; the higher level of CSFoligosaccharides, and the lower a total equivalent age in the Memory Screening ( $p=0.039$ (age: $p=0.136$ ) [23].

The significantly better performance of subgroup 3 patients in BOT-2 subtests (Upper limb coordination and Balance), may indicate a genotype-phenotype correlation concerning balance and muscular coordination. Disabilities in muscular coordination and balance are well known and important clinical features [1, 34], which makes a genotype-phenotype correlation concerning these

Table 3 The distribution of patients in the three subgroups

\begin{tabular}{|c|c|c|c|c|c|}
\hline Genotype/subcellular localisation subgroups & $\mathrm{N}$ & Age +/-SD, Mean (years) & Age Median (years) & Age Minimum (years) & Age Maximum (years) \\
\hline Subgroup 1 & 21 & $22.9 \pm 11.6$ & 23.0 & 5.7 & 42.1 \\
\hline \multicolumn{6}{|l|}{$\begin{array}{l}\text { Two null-mutations } \\
\text { (nonsense, frameshift, large truncation) }\end{array}$} \\
\hline Subgroup 2 & 32 & $17.9 \pm 7.6$ & 17.9 & 6.0 & 35.0 \\
\hline \multicolumn{6}{|l|}{$\begin{array}{l}\text { Missense/in-frame dup/del localised } \\
\text { to the ER }\end{array}$} \\
\hline Subgroup 3 & 13 & $17.4 \pm 7.8$ & 17.3 & 5.4 & 29.0 \\
\hline \multicolumn{6}{|l|}{$\begin{array}{l}\text { Missense/in-frame dup/del } \\
\text { localised to the lysosomes }\end{array}$} \\
\hline Total & 66 & $19.4 \pm 9.3$ & 17.3 & 5.4 & 42.1 \\
\hline
\end{tabular}




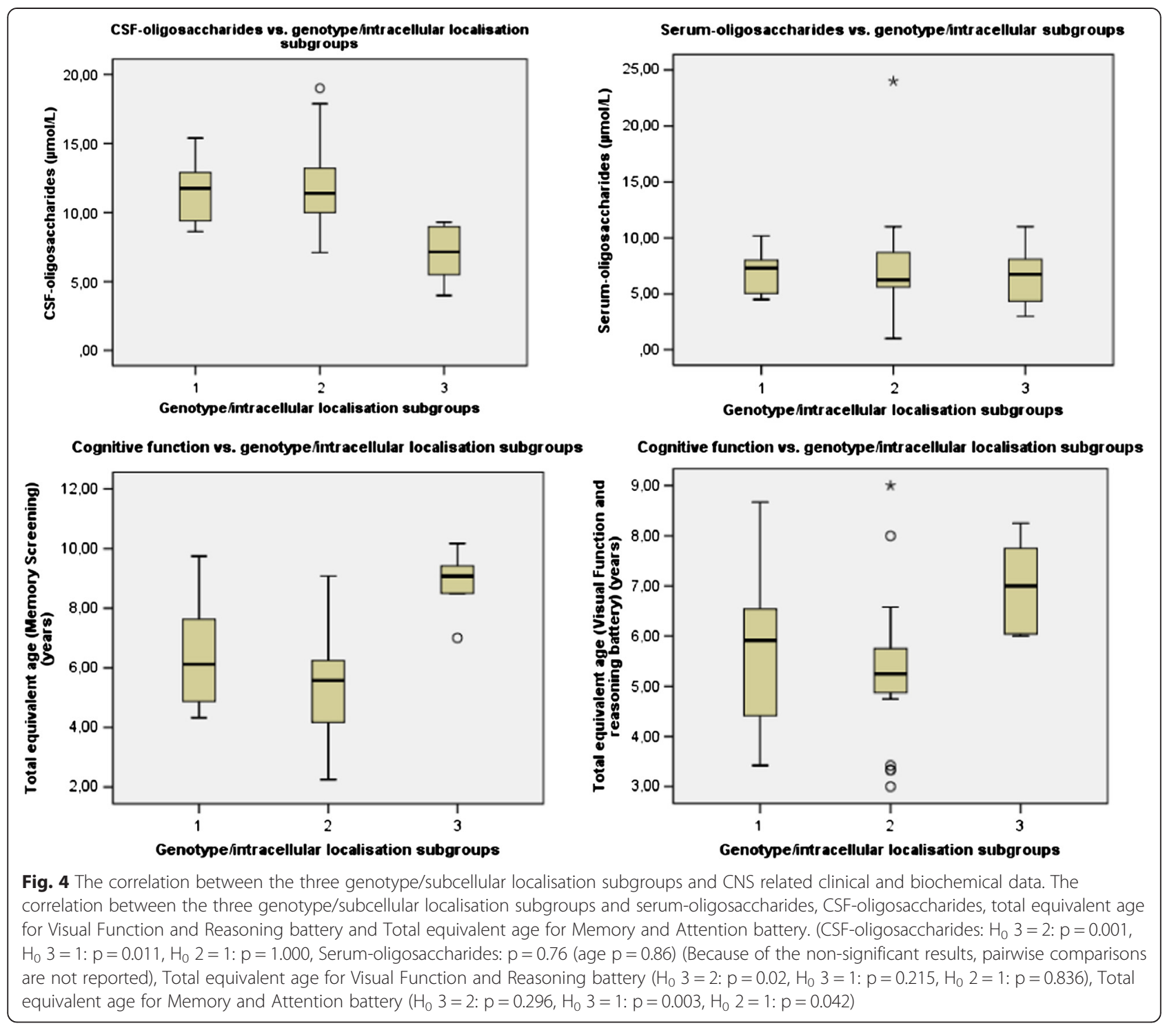

skills particularly interesting. A tendency to a genotypephenotype correlation concerning the 6-MWT was also found, which might indicate a relationship between the different genotype/subcellular localisation subgroups and motor function in general.

Due to intellectual disabilities, correctly performed spirometry has been a challenge for some alpha-mannosidosis patients. Thus, the values given at spirometry may be inaccurate for some of the patients $[17,18]$ and the relationship between the $\mathrm{FVC} \%$ and the different subgroups may be uncertain.

No significant correlation between genotype/subcellular localisation subgroups and lysosomal alpha-mannosidase residual activity was found, though previous studies have suggested that severity of clinical expression is related to the degree of reduced enzyme activity in human alphamannosidosis patients [35]. However, other studies are in accordance with our results $[7,36]$. It has been suggested that the missing correlation might be due to alpha-mannosidases from other cellular compartments that confer residual activity at low $\mathrm{pH}$. This may be misinterpreted as residual lysosomal alpha-mannosidase activity [16]. Moreover, mutant MAN2B1 proteins, mislocalised to non-lysosomal compartments (and thus incapable of lysosomal glycoprotein degradation), may show some activity when released into serum from leukocytes [7].

Measuring residual lysosomal alpha-mannosidase activity is difficult. The values for alpha-mannosidase activities are low in all patients; hence, even minor potential sources of error may influence the results significantly and obscure a genotype/phenotype correlation. Development of more accurate methods is necessary for final conclusions of a genotype-phenotype 


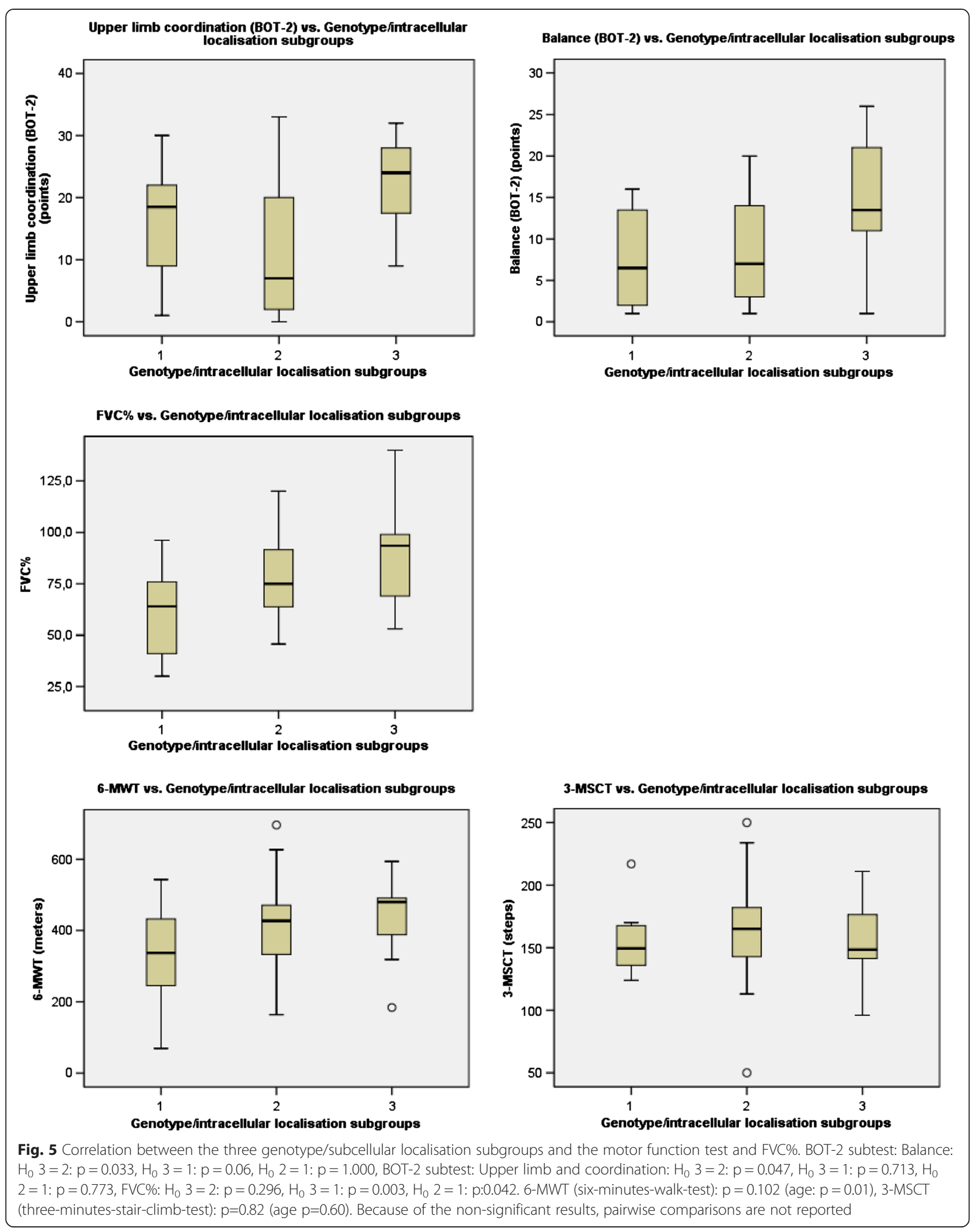


Table 4 Correlations between the three genotype/subcellular localisation subgroups and biochemical and clinical test results

\begin{tabular}{|c|c|c|c|c|c|c|c|c|c|}
\hline Analysis/clinical test & $\mathrm{N}$ & Subgr. 1, mean & Subgr. 2, mean & Subgr. 3, mean & Min. & Max. & Total, mean & SD & p-value Pairwise comparison* \\
\hline \multirow[t]{2}{*}{ CSF-oligo $(\mu \mathrm{mol} / \mathrm{L})$} & \multirow[t]{2}{*}{$35 / 35$} & \multirow[t]{2}{*}{$11.5 \pm 2.3$} & \multirow[t]{2}{*}{$11.9 \pm 3.2$} & \multirow[t]{2}{*}{$7.1 \pm 2.0$} & \multirow[t]{2}{*}{4.0} & \multirow[t]{2}{*}{19} & \multirow[t]{2}{*}{10.7} & \multirow[t]{2}{*}{3.4} & $H_{0} 3=2: p=0.001, H_{0} 3=1: p=0.011$ \\
\hline & & & & & & & & & $H_{0} 2=1: p=1.000$ \\
\hline \multirow{2}{*}{$\begin{array}{l}\text { Cognitive test } \\
\text { (VR battery) (years) }\end{array}$} & \multirow[t]{2}{*}{$35 / 35$} & \multirow[t]{2}{*}{$5.8 \pm 1.7$} & \multirow[t]{2}{*}{$5.3 \pm 1.5$} & \multirow[t]{2}{*}{$7.0 \pm 0.9$} & \multirow[t]{2}{*}{3.0} & \multirow[t]{2}{*}{9.0} & \multirow[t]{2}{*}{5.8} & \multirow[t]{2}{*}{1.6} & $H_{0} 3=2: p=0.049, H_{0} 3=1: p=0.344$ \\
\hline & & & & & & & & & $H_{0} 2=1: p=1.000$ \\
\hline \multirow{2}{*}{$\begin{array}{l}\text { Cognitive test } \\
\text { (Memory Screening) (y) }\end{array}$} & \multirow[t]{2}{*}{$26 / 26$} & \multirow[t]{2}{*}{$6.9 \pm 1.9$} & \multirow[t]{2}{*}{$5.4 \pm 2.0$} & \multirow[t]{2}{*}{$8.8 \pm 1.2$} & \multirow[t]{2}{*}{2.3} & \multirow[t]{2}{*}{10.2} & \multirow[t]{2}{*}{6.5} & \multirow[t]{2}{*}{2.3} & $H_{0} 3=2: p=0.02, H_{0} 3=1: p=0.215$ \\
\hline & & & & & & & & & $H_{0} 2=1: p=0.836$ \\
\hline \multirow[t]{2}{*}{ FVC\% (\%) } & \multirow[t]{2}{*}{$54 / 66^{*}$} & \multirow[t]{2}{*}{$62.3 \pm 20.7$} & \multirow[t]{2}{*}{$77.5 \pm 19 \%$} & \multirow[t]{2}{*}{$90.7 \pm 22.7$} & \multirow[t]{2}{*}{30} & \multirow[t]{2}{*}{140} & \multirow[t]{2}{*}{75.2} & \multirow[t]{2}{*}{22.7} & $H_{0} 3=2: p=0.296, H_{0} 3=1: p=0.003$ \\
\hline & & & & & & & & & $H_{0} 2=1: p=0.042$ \\
\hline \multirow[t]{2}{*}{ BOT-2 (Coor.) (points) } & \multirow[t]{2}{*}{$35 / 35$} & $16.3 \pm 10.1$ & $11.3 \pm 10.8$ & $22.5 \pm 7.6$ & 1 & 33 & 15 & 10.8 & $H_{0} 3=2: p=0.047, H_{0} 3=1: p=0.713$ \\
\hline & & & & & & & & & $H_{0} 2=1: p=0.773$ \\
\hline BOT-2 (Balance) (points) & $35 / 35$ & $7.6 \pm 6.2$ & $8.4 \pm 6.1$ & $14.8 \pm 7.9$ & 1 & 26 & 9.7 & 7.0 & $H_{0} 3=2: p=0.033, H_{0} 3=1: p=0.06$ \\
\hline & & & & & & & & & $H_{0} 2=1: p=1.00$ \\
\hline
\end{tabular}

Significant correlations between the three genotype/subcellular localisation subgroups and CSF-oligosaccharides (CSF-oligo), Cognitive test (VR battery) (=Total equivalent age for the Visual function and reasoning battery), Cognitive test (Memory Screening) (Total equivalent age for the Memory Screening), Forced Vital Capacity (FVC\%), BOT-2 (Upper limb coordination) (Coor.) and BOT-2 (Balance)

$\mathrm{N}=$ number, $\min .=$ minimum, Max. $=$ Maximum, $\mathrm{SD}=$ standard deviation, ${ }^{*} \mathrm{H}_{0} 3=2 / \mathrm{H}_{0} 3=1 / \mathrm{H}_{0} 2=1$ : Refers to the null hypothesis $\left(\mathrm{H}_{0}\right)$ for the comparison of subgroup 3 versus subgroup $2 /$ subgroup 3 versus subgroup 1 / subgroup 2 versus subgroup 1. $\mathrm{p}<0.05$ rejects the $\mathrm{H}_{0}$

*54/66 were able to perform a spirometry 
correlation concerning residual lysosomal alphamannosidase activity.

Previously, genotype-phenotype correlations in alphamannosidosis have been investigated in two studies [11, 16]. Our findings are not in line with those of Berg et al. [16] and Riise Stensland et al. [11], who found no apparent genotype-phenotype correlation when investigating the relationship between different types of mutations and the clinical subtype classification in 23 and 130 patients, respectively. These studies classified the patients into three phenotypic subgroups, type 1, type 2 and type 3 , according to the severity of manifestations as suggested by Malm and Nilssen [34, 37]. Both studies were conducted at a time before proper clinical end-points were established. A challenge using these three phenotypic subgroups is that most of the patients diagnosed with alpha-mannosidosis are intermediate type 2 patients. In the study by Riise Stensland et al. [11], 106 of 130 patients were classified as type 2, and in Berg et al. [16], the clinical data were not collected in a standardised format, but 22 of 23 patients were described with moderate clinical manifestations.

Our cohort has not been classified into the above three phenotypic subtypes, since the disease manifestations display a continuum of clinical severity, as documented from our large cohort of alpha-mannosidosis patients and newer literature [1]. Instead, individual results from clinical and biochemical tests were used to investigate the relationship with the three genotype/subcellular localisation subgroups, making a more detailed and precise evaluation possible.

Our data have been collected in a standardised manner. For rhLAMAN-02 and rhLAMAN-05 the same doctor/nurse/ physiotherapist etc. assisted the patients when performing the tests. For the two previous studies $[11,16]$, clinical information for most of the patients was collected by the referring physicians, which could possibly introduce dissimilarity in the reporting.

Our findings demonstrated that genotypes allowing mutant MAN2B1 protein to enter the lysosomes, correlated positively with several clinical and biochemical parameters, though further investigation of a larger cohort will be necessary before such correlation can be used in clinical practice for postnatal or prenatal prognostics. Despite our findings challenges still remain.

One challenge is a phenotypic variation within sibships with identical MAN2B1 mutations [12-15, 38]. Riise Stensland et al. [11] described 16 sibships with alphamannosidosis classified in phenotypic subgroups 1-3, and one was discordant. Among our patients participating in rhLAMAN-02 or rhLAMAN-05, one of three sibships presented with different degrees of disease involvement, especially with regard to the cognitive function, though both patients in this sibship belonged to the attenuated type 2 . The most affected sibling in this sibship was treated twice with protracted mechanical ventilation during the first 1.5 years of life, due to Respiratory Syncytial Virus and bacterial pneumonia, which may have influenced the severity of the phenotype, including the cognitive function. Based on our and Riise Stensland et al.'s findings, the phenotypic variation between sibs might not be as frequent as previously reported, and may be explained by environmental and/or epigenetic factors.

Another challenge is the fact that some patients clinically characterised as less severely or moderately affected are homozygous for null mutations [16]. A possible explanation to this phenomenon is that other mannosidases than lysosomal alpha-mannosidase, may contribute to salvage pathways in the degradation of glycoproteins. Evidence for a role of cytosolic alpha-mannosidase in the subcellular degradation of oligosaccharides was provided by Grard et al. [39, 40]. Hence, it is possible that allelic heterogeneity among extra-lysosomal alpha-mannosidases could influence the phenotypic expression of alphamannosidosis.

In view of the above considerations, we believe that the genotype is fundamental in determining the severity of alpha-mannosidosis, but other factors, both genetic, epigenetic and environmental, may contribute to the observed clinical variation. As examples, occurrence of severe infections (which is common in alphamannosidosis), differences in education, motor and cognitive stimulation may affect the phenotype.

\section{Conclusion}

In conclusion, this study is the first to investigate the genotype-phenotype correlation in alpha-mannosidosis by classifying the cohort into three genotypic/subcellular localisation subgroups and investigating the correlation between the subgroups and the individual results of clinical and biochemical tests. Our data indicate a genotypephenotype relationship between the genotype/subcellular localisation subgroups and the cognitive function, the BOT-2 motoric test, FVC\% and the storage of oligosaccharides in CSF. Patients from subgroup 3, with at least one mutation that allows localisation of the mutant MAN2B1 protein to the lysosomes, performed significantly better and had less abnormal results in some of the clinical tests and biochemical analyses, compared to patients in subgroup 2 and/or subgroup 1 .

Further investigation of a larger cohort will be necessary before such correlation can be used in clinical practice for postnatal or prenatal prognostics.

\section{Details of ethics approvals}

This study includes baseline data from rhLAMAN-01, rhLAMAN-02 and rhLAMAN-05. All three studies were 
approved by the local Ethics Committee and additionally rhLAMAN-02 and rhLAMAN-05 were approved by the Danish Medicines Agency.

EudraCT numbers: 2010-022084-36 (NCT00498420) and 2012-000979-17 (NCT01681953).

\begin{abstract}
Abbreviations
3MSCT: Three-minute Stair Climb Test; 6MWT: Six-minute Walk Test: ATS: American thoracic society; BOT2: Bruininks-Oseretsky test of Motor Proficiency; CNS: Central Nervous System; CSF: Cerebrospinal Fluid; ER: Endoplasmic reticulum; ERT: Enzyme Replacement Therapy; FVC: Forced Vital Capacity; GCP: Good Clinical Practice; GFAp: Glial Fibrillary Acidic protein; Leiter R: Leiter International Performance Scale-Revised; NFLp: Neurofilament Protein; PFT: Pulmonary Function Testing; PTA: Pure Tone Audiometry; T-tau: Tau Protein; VUS: Variant of unknown clinical significance; WT: Wild-type.
\end{abstract}

\section{Competing interests}

Zymenex provides the investigational recombinant human enzyme Lamazym (rhLAMAN) tested in the clinical trials rhLAMAN-02 and rhLAMAN-05. The investigators or any other study-site personnel are not employed by Zymenex. Dr. Line Borgwardt has received reimbursement of travel expenses for a scientific meeting by Zymenex. No other reimbursements/fees/funds/salaries have been accepted. The authors; except Fogh I who is employed at Zymenex; confirms independence from the sponsors; the content of the article has not been influenced by the sponsors.

\section{Authors' contribution}

LB: Contributed to the design, acquisition of data, analyses and interpretation of data, preparation of the manuscript. HMFRS: Contributed to the design, acquisition of data, analyses and interpretation of data, preparation of the manuscript. KJO: Contributed to the analyses and interpretation of data, critical review of the manuscript. FW: Contributed to the analyses and interpretation of data, critical review of the manuscript. HBK: Contributed to the acquisition of data. MB: Contributed to the acquisition of data, critical review of the manuscript. YM: Contributed to the acquisition of data, critical review of the manuscript. LA: Contributed to the acquisition of data, critical review of the manuscript. JF: Contributed to the design, interpretation of data, critical review of the manuscript. $\varnothing \mathrm{N}$ : Contributed to the design, analyses and interpretation of data, preparation of the manuscript. CID: Contributed to the design, acquisition of data, critical review of the manuscript. AML: Contributed to the design, analyses and interpretation of data, preparation of the manuscript. All authors read and approved the final manuscript.

\section{Acknowledgements}

We want to express a special thanks to the patients and their families for their participation and dedicated efforts in the studies. We thank our study-site personnel Marianne Luiten, Sannie Mahncke, Pernille Strøm, Camilla Sørensen, Lærke Freiberg, Nanna Kjær, Josefine Gutte Koch, Kamille Fogh; Pia Ringholm and Susanne Rodholm, Lene Skammelsen, Zymenex A/S, Hillerød, Denmark; Torben Balchen and Jesper Sonne, including their personnel, ZeloDantrial A/S, Copenhagen, Denmark. We thank Vigdis Brox for help with mutation analyses. We thank EU-framework 6 and 7 programs for supporting the HUEMAN and the ALPHA-MAN projects economically. We thank the entire HUEMAN and the ALPHA-MAN consortium and the following physicians: Lars Holme Nielsen, Steen Østergaard Olsen, Jesper Steensberg, Kim G Nielsen, Else Danielsen, Carsten Thomsen, Ans van der Ploeg, Hannerieke van den Hout, Frits Wijburg, Encarna Guillén-Navarro, Christopher Lindberg and Birgitte Hanel, Anne-Marie Thuesen, Ulla Haugsted, Thea Pearl, Anne Sylvest Olsen, Kirsten Glarborg. A last we thank Flemming Skovby, Department of Clinical Genetics, Rigshospitalet, Copenhagen for critical review of the manuscript.

\section{Funding}

This work was supported by the HUE-MAN consortium (European Commission FP VI Contract LSHM-CT-2006-018692) and the ALPHA-MAN consortium (European Commission FP VII Contract HEALTH.20102.4.4-1).

\section{Author details}

'Department of Clinical Genetics, Centre for Inherited Metabolic Diseases, Copenhagen University Hospital Rigshospitalet, 9 Blegdamsvej, 2100 Copenhagen, Denmark. ${ }^{2}$ Department of Medical Genetics, Division of Child and Adolescent Health, University Hospital of North Norway, Tromsø, Norway. ${ }^{3}$ Larix, CRO, Ballerup, Denmark. ${ }^{4}$ Zentrum für Kinder - und Jugendmedizin, Villa Metabolica, Mainz, Germany. ${ }^{5}$ Zymenex A/S (Chiesi Group), Hilleroed, Denmark. ${ }^{6}$ Department of Clinical Medicine, Medical Genetics, University of North Nonway, Tromsø, Norway.

Received: 9 February 2015 Accepted: 19 May 2015

Published online: 06 June 2015

\section{References}

1. Malm D, Nilssen $\varnothing$. Alpha-mannosidosis. GeneReviews: NCBI Bookshelf; 2012 [www.ncbi.nlm.nih.gov/books/NBK1396/]

2. Borgwardt L, Dali C, Lund A. Alpha-mannosidosis - a review of genetic, clinical findings and options of treatment. Pediatr Endocrinol Rev. 2014;12 Suppl 1:185-91.

3. H.H.Freeze: Degradation and Turnover of Glycans. In Essentials of Glycobiology. 2nd edition edition. Edited by Ajit Varki. Cold Spring Harbor Perspectives in Biology; 1999.

4. Thomas GH. Disorder of Glycoprotein Degradation. The Metabolic \&Molecular Bases of Inherited Disease; McGraw-Hill, Medical Publishing Division, 2001.

5. HUGO Gene Nomenclature Committee. [http://www.genenames.org/]

6. Riise Stensland H, Frantzen G, Kuokkanen E, Buvang EK, Klenow HB, Heikinheimo P, et al. Amamutdb.no: a relational database for MAN2B1 allelic variants that compiles genotypes, clinical phenotypes, and biochemical and structural data of mutant MAN2B1 in a-mannosidosis. Hum Mutat. 2015;36(6):581-6.

7. Nilssen $\varnothing$, Berg T, Riise HM, Ramachandran U, Evjen G, Hansen GM, et al. alpha-Mannosidosis: functional cloning of the lysosomal alpha-mannosidase CDNA and identification of a mutation in two affected siblings. Hum Mol Genet. 1997;6:717-26.

8. Hansen G, Berg T, Riise Stensland HM, Heikinheimo P, Klenow H, Evjen G, et al. Intracellular transport of human lysosomal alpha-mannosidase and alpha-mannosidosis-related mutants. Biochem J. 2004;381:537-46.

9. Kuokkanen E, Riise Stensland HM, Smith W, Kjeldsen BE, Van NL, Nilssen O, et al. Molecular and cellular characterization of novel \{alpha\}-mannosidosis mutations. Hum Mol Genet. 2011;20:2651-61.

10. HGMD Professional 2015.1 [(https:/www.portal.biobase-international.com/ hgmd/pro/gene.php?gene=MAn2B1//]

11. Riise Stensland HM, Klenow HB, Van NL, Hansen GM, Malm D, Nilssen Ø. Identification of 83 novel alpha-mannosidosis-associated sequence variants: functional analysis of MAN2B1 missense mutations. Hum Mutat. 2012;33:511-20.

12. Ara JR, Mayayo E, Marzo ME, Guelbenzu S, Chabas A, Pina MA, et al. Neurological impairment in alpha-mannosidosis: a longitudinal clinical and MRI study of a brother and sister. Childs Nerv Syst. 1999;15:369-71.

13. Govender R, Mubaiwa L. Alpha-mannosidosis: a report of 2 siblings and review of the literature. J Child Neurol. 2013;29(1):131-4.

14. Michelakakis H, Dimitriou E, Mylona-Karayanni C, Bartsocas CS. Phenotypic variability of mannosidosis type II: report of two Greek siblings. Genet Couns. 1992;3:195-9.

15. Mitchell ML, Erickson RP, Schmid D, Hieber V, Poznanski AK, Hicks SP. Mannosidosis: two brothers with different degrees of disease severity. Clin Genet. 1981;20:191-202.

16. Berg T, Riise HM, Hansen GM, Malm D, Tranebjaerg L, Tollersrud OK, et al. Spectrum of mutations in alpha-mannosidosis. Am J Hum Genet. 1999;64:77-88.

17. Borgwardt L, Dali Cl, Fogh J, Mansson JE, Olsen KJ, Beck HC, et al. Enzyme replacement therapy for alpha-mannosidosis: 12 months follow-up of a single centre, randomised, multiple dose study. J Inherit Metab Dis. 2013;36(6):1015-24.

18. Beck M, Olsen KJ, Wraith JE, Zeman J, Michalski JC, Saftig P, et al. Natural history of alpha mannosidosis a longitudinal study. Orphanet J Rare Dis. 2013:8:88.

19. Blennow K, Wallin A, Agren H, Spenger C, Siegfried J, Vanmechelen E. Tau protein in cerebrospinal fluid: a biochemical marker for axonal degeneration in Alzheimer disease? Mol Chem Neuropathol. 1995;26:231-45. 
20. Rosengren LE, Ahlsen G, Belfrage M, Gillberg C, Haglid KG, Hamberger A. A sensitive ELISA for glial fibrillary acidic protein: application in CSF of children. J Neurosci Methods. 1992;44:113-9.

21. Rosengren LE, Karlsson JE, Karlsson JO, Persson LI, Wikkelso C. Patients with amyotrophic lateral sclerosis and other neurodegenerative diseases have increased levels of neurofilament protein in CSF. J Neurochem. 1996;67:2013-8.

22. Masson PK, Lundblad A. Mannosidosis: detection of the disease and of heterozygotes using serum and leucocytes. Biochem Biophys Res Commun. 1974;56:296-303.

23. Borgwardt L, Thuesen A, Olsen K, Fogh J, Dali C, Lund A. Cognitive profile and activities of daily living: 35 patients with alpha-mannosidosis, J Inherit Metab Dis 2015; In press.

24. Tsatsanis KD, Dartnall N, Cicchetti D, Sparrow SS, Klin A, Volkmar FR. Concurrent validity and classification accuracy of the Leiter and Leiter-R in low-functioning children with autism. J Autism Dev Disord. 2003;33:23-30.

25. Myrelid A, Bergman S, Elfvik SM, Jonsson B, Nyberg F, Gustafsson J, et al. Late effects of early growth hormone treatment in down syndrome. Acta Paediatr. 2010;99:763-9.

26. Colletti L, Zoccante L. Nonverbal cognitive abilities and auditory performance in children fitted with auditory brainstem implants: preliminary report. Laryngoscope. 2008;118:1443-8.

27. Beydon N, Davis SD, Lombardi E, Allen JL, Arets HG, Aurora P, et al. An official American Thoracic Society/European Respiratory Society statement: pulmonary function testing in preschool children. Am J Respir Crit Care Med. 2007;175:1304-45.

28. ATS committee on profiency standards for clinical pulmonary function laboratories. ATS statement: guidelines for the six-minute walk test. Am J Respir Crit Care Med. 2002;166:111-7.

29. Bolton JW, Weiman DS, Haynes JL, Hornung CA, Olsen GN, Almond CH. Stair climbing as an indicator of pulmonary function. Chest. 1987;92:783-8.

30. Harmatz P, Giugliani R, Schwartz IV, Guffon N, Teles EL, Miranda MC, et al. Long-term follow-up of endurance and safety outcomes during enzyme replacement therapy for mucopolysaccharidosis Vl: final results of three clinical studies of recombinant human $\mathrm{N}$-acetylgalactosamine 4-sulfatase. Mol Genet Metab. 2008;94:469-75.

31. Holden DA, Rice TW, Stelmach K, Meeker DP. Exercise testing, 6-min walk, and stair climb in the evaluation of patients at high risk for pulmonary resection. Chest. 1992;102:1774-9.

32. Deitz JC, Kartin D, Kopp K. Review of the Bruininks-Oseretsky test of motor proficiency, second edition (BOT-2). Phys Occup Ther Pediatr. 2007;27:87-102.

33. ISO 8253-1 and BS EN ISO 8253-2 Standards for Acoustic Audiometric Test Methods (updated in 1998) and Department of Health (1994) Building Note 12, Supp 3 ENT and Audiology Clinics/Hearing Aid Centres, NHS Estates. 1998. [www.ndcs.org.uk/document.rm?id=6247]

34. Malm D, Riise Stensland HM, Edvardsen $\varnothing$, Nilssen $\varnothing$. The natural course and complications of alpha-mannosidosis-a retrospective and descriptive study. J Inherit Metab Dis. 2014;37:79-82.

35. Bennet JK, Dembure PP, Elsas LJ. Clinical and biochemical analysis of two families with type I and type II mannosidosis. Am J Med Genet. 1995:55:21-6.

36. Berg T, Healy PJ, Tollersrud OK, Nilssen $\varnothing$. Molecular heterogeneity for bovine alpha-mannosidosis: PCR based assays for detection of breedspecific mutations. Res Vet Sci. 1997;63:279-82

37. Malm D, Nilssen $\varnothing$. Alpha-mannosidosis. Orphanet J Rare Dis. 2008;23:3-21.

38. Malm D, Tollersrud OK, Tranebjaerg L, Mansson JE. Alpha-mannosidosis. Tidsskr Nor Laegeforen. 1995;115:594-7.

39. Grard T, Herman V, Saint-Pol A, Kmiecik D, Labiau O, Mir AM, et al. Oligomannosides or oligosaccharide-lipids as potential substrates for rat liver cytosolic alpha-D-mannosidase. Biochem J. 1996:316(Pt 3):787-92.

40. Paciotti S, Persichette E, Kelin K, Tasegian A, Duvet S, Harmann D, et al. Accumulation of free oligosaccharides and tissue damage in cytosolic a-mannosidase (Man2c1)-deficient mice. J Biol Chem. 2014;289:9611-22.

\section{Submit your next manuscript to BioMed Central and take full advantage of:}

- Convenient online submission

- Thorough peer review

- No space constraints or color figure charges

- Immediate publication on acceptance

- Inclusion in PubMed, CAS, Scopus and Google Scholar

- Research which is freely available for redistribution

Submit your manuscript at www.biomedcentral.com/submit 